Relations industrielles

Industrial Relations

\title{
Lallement, Michel, Sociologie des relations professionnelles
}

\section{Gilles Laflamme}

Volume 51, numéro 4, 1996

URI : https://id.erudit.org/iderudit/051143ar

DOI : https://doi.org/10.7202/051143ar

Aller au sommaire du numéro

\section{Éditeur(s)}

Département des relations industrielles de l'Université Laval

\section{ISSN}

0034-379X (imprimé)

1703-8138 (numérique)

Découvrir la revue

Citer ce compte rendu

Laflamme, G. (1996). Compte rendu de [Lallement, Michel, Sociologie des relations professionnelles]. Relations industrielles / Industrial Relations, 51(4), 853-853. https://doi.org/10.7202/051143ar

Tous droits réservés (C) Département des relations industrielles de l'Université Laval, 1996
Ce document est protégé par la loi sur le droit d'auteur. L’utilisation des services d'Érudit (y compris la reproduction) est assujettie à sa politique d'utilisation que vous pouvez consulter en ligne.

https://apropos.erudit.org/fr/usagers/politique-dutilisation/ 


\section{Sociologie des relations professionnelles}

par Michel LALlEMENT, Paris, Éditions La Découverte, 1996, 123 p., ISBN 27071-2522-4.

Cet ouvrage de Michel Lallement constitue une vue d'ensemble sur les relations professionnelles et c'était une véritable gageure que de pouvoir présenter, en si peu de pages, avec cohérence et intérêt, dans une perspective comparative, l'édification des relations professionnelles (chapitre I) et l'interprétation de leur origine et de leur développement (chapitre II), les déterminants des identités et des stratégies des acteurs qui animent les systèmes de relations professionnelles contemporains (chapitre III) ainsi que les modes de gestion des conflits (chapitre IV). Enfin, comme on ne saurait traiter des relations professionnelles, surtout dans la période de crise que l'on connait actuellement, sans discuter de leur impact sur la régulation des marchés du travail, l'auteur, dans un dernier chapitre (chapitre V), après avoir mis en évidence le rôle et la diversité des formes de régulation du marché du travail, dégage la nature des liens multiples et évolutifs entre les relations professionnelles et la régulation des marchés du travail.

Michel Lallement n'a pas cherché à développer, de façon exhaustive, ni le développement du syndicalisme dans les pays visés par son ouvrage (particulièrement la France, l'Angleterre, le Japon, la Suède, l'Allemagne et les États-Unis), ni les logiques qui ont conduit à la construction des systèmes de relations professionnelles pour chacun de ces pays. Néanmoins, il a su, pour chacun des systèmes, dégager les variables essentielles qui nous permettent de saisir, dans le temps et dans l'espace, le rôle régulateur des divers systèmes de même que leur impact, par le jeu des acteurs, dans la production et la transformation des règles de fonctionnement des marchés du travail.

Les spécialistes des relations professionnelles retrouveront dans cet ouvrage une présentation un peu trop sommaire, et absente d'une certaine perspective critique, des auteurs classiques qui ont marqué non seulement le domaine de recherches que constituent les relations professionnelles, mais encore l'exercice d'une profession. Cela est particulièrement vrai pour ceux et celles qui sortent des grandes institutions universitaires nordaméricaines. Toutefois, compte tenu des objectifs poursuivis dans cet ouvrage, il était fort difficile pour l'auteur de se livrer à une analyse critique des diverses théories et approches qui ont cherché à expliquer tant la naissance et le développement du syndicalisme que la construction même des rapports ayant pour objet de réguler les tensions entre les acteurs sociaux. Cette diversité des approches, des éléments d'interprétation est omniprésente tout au long de cet ouvrage. Le seul regret est que l'auteur présente chacune d'elles de façon trop succincte.

Bref, il s'agit d'un ouvrage intéressant qui permet de suivre l'évolution des assises institutionnelles de systèmes de relations professionnelles de même que les mutations syndicales qui se sont opérées de la fin du XIX ${ }^{e}$ siècle à aujourd'hui. Pour le lecteur qui souhaiterait un traitement plus nuancé de certaines questions fondamentales, la richesse des repères bibliographiques lui servira de guide.

GLLES LAFLAMME Université Laval 